\title{
Nanoribbons with Non-Alternant Topology from Fusion of Polyazulene: Carbon Allotropes Beyond Graphene
}

\author{
Qitang Fan $^{1 \ddagger}$, Daniel Martin-Jimenez ${ }^{2,3 \ddagger}$, Daniel Ebeling ${ }^{2,3}$, Claudio K. Krug ${ }^{1}$, Lea Brechmann ${ }^{1}$, Corinna \\ Kohlmeyer ${ }^{4}$, Gerhard Hilt ${ }^{4}$, Wolfgang Hieringer ${ }^{5}$, André Schirmeisen ${ }^{2,3}$, J. Michael Gottfried ${ }^{1}$ \\ ${ }^{1}$ Department of Chemistry, Philipps University Marburg, Hans-Meerwein-Straße 4, 35032 Marburg, Germany \\ ${ }^{2}$ Institute of Applied Physics (IAP), Justus Liebig University Gießen, Heinrich-Buff-Ring 16, 35392 Gießen, Germany \\ ${ }^{3}$ Center for Materials Research (LaMa), Justus Liebig University Giessen, Heinrich-Buff-Ring 16, 35392 Giessen, \\ Germany \\ ${ }^{4}$ Institute of Chemistry, Carl von Ossietzky University Oldenburg, Carl-von-Ossietzky-Straße 9-11, 26111 Oldenburg, \\ Germany \\ ${ }^{5}$ Theoretical Chemistry and Interdisciplinary Center for Molecular Materials (ICMM), Department of Chemistry and \\ Pharmacy, Friedrich-Alexander-Universität Erlangen-Nürnberg, Egerlandstraße 3, 91058 Erlangen, Germany
}

\begin{abstract}
Various two-dimensional (2D) carbon allotropes with non-alternant topologies, such as pentaheptites and phagraphene, have been proposed. Predictions indicate that these metastable carbon polymorphs, which contain odd-numbered rings, possess unusual (opto)electronic properties. However, none of these materials has been achieved experimentally due to synthetic challenges. In this work, by using on-surface synthesis, nanoribbons of the non-alternant graphene allotropes, phagraphene and tetra-pentahepta(TPH)-graphene have been obtained by dehydrogenative C-C coupling of 2,6-polyazulene chains. These chains were formed in a preceding reaction step via on-surface Ullmann coupling of 2,6dibromoazulene. Low-temperature scanning probe microscopies with CO-functionalized tip and density functional theory calculations have been used to elucidate their structural properties. The proposed synthesis of non-alternant carbon nanoribbons from the fusion of synthetic line-defects may pave the way for large-area preparation of novel 2D carbon allotropes.
\end{abstract}




\section{INTRODUCTION}

Modifying the topology of pristine graphene with atomic precision has attracted tremendous attention in recent years, because it serves as a versatile route to novel graphene-related materials with exotic (opto)electronic properties. Examples include graphene nanoribbons, ${ }^{1-5}$ porous graphene, ${ }^{6-8}$ and nanographenes, ${ }^{9-13}$ which have been successfully prepared through various bottom-up approaches. The majority of these materials are cut-outs of graphene, i.e., they are composed of $\mathrm{sp}^{2}$ carbon atoms arranged to form hexagonal rings. The electronic properties of these so-called alternant $\pi$-electron systems ${ }^{14}$ are determined by factors such as size, shape, and edge structure. An alternative and not yet well-developed method to modify graphene is to change the connectivity pattern of the $\mathrm{sp}^{2}$ carbon atoms in a non-hexagonal manner. Theoretical considerations have predicted the existence of numerous planar non-hexagonal carbon allotropes ${ }^{15-17}$, including pentaheptites (1, Fig. 1a), ${ }^{18-20}$ haeckelites, ${ }^{21}$ T-graphene, ${ }^{22}$ octa-penta graphene, ${ }^{23}$ pentahexoctites, ${ }^{24}$ and phagraphene $2 .{ }^{25}$ These $2 \mathrm{D}$ carbon allotropes are expected to show dramatically different properties regarding electron transportation, ${ }^{26,27}$ magnetism, ${ }^{28}$ chemical reactivity ${ }^{29}$ and mechanical stability. ${ }^{28,30,31}$ However, the experimental realization of these materials lags far behind the theoretical studies. This problem is mainly caused by the enormous challenges that lie in the atomically precise control over the growth of extended non-hexagonal 2D carbon structures. Although non-hexagonal structural motifs, such as the Stone-Wales (5-7-7-5) and 5-8-5 defects, can be randomly introduced into graphene by moderate electron beam bombardment, irradiation or chemical treatment, ${ }^{32,}$ ${ }^{33}$ the periodic arrangement of these structural elements is so far difficult to achieve.

Very recently, the on-surface synthetic approach has demonstrated its potential for the growth of nonhexagonal 2D carbon allotropes in a more controlled way. Through cyclodehydrogenative C-C coupling of specially designed benzenoid organic molecules, it was possible to make nanographenes with embedded 5-7 ring pairs ${ }^{11,34-36}$ and graphene-like carbon nanoribbons with periodically arranged 4-8-4 motifs..$^{37,38}$ The growth of polymers with periodically arranged odd-numbered polygon motifs, e.g., the 5-7 ring pairs, is intriguing because the connectivity pattern of carbon atoms alters from alternant (hexagons) into nonalternant. ${ }^{14}$ In contrast, the 4-8 structural motif is non-hexagonal, but remains alternant. The former case is especially interesting, because molecular $\pi$-electron systems with non-alternant topology have drastically different electronic properties compared to their alternant counterparts. ${ }^{14,39}$ All the above mentioned examples of growing 5-7 motifs represent only embedded point defects. Up to now, the embedment of a continuous one-dimensional (1D) arrangement of pentagon-heptagon (5-7) motifs is still lacking.

Here, by employing 2,6-dibromoazulene (4, Fig. 1b) as a non-alternant precursor consisting of annulated 5- and 7-membered rings, we have successfully obtained the 2,6-polyazulene chains 5 via on-surface Ullmann coupling. The 2,6-polyazulene chain could be reckoned as 1D line defect with fused pentagons and heptagons (5-7 motif). Subsequently, the lateral dehydrogenative coupling (fusion) of two 2,6polyazulene chains results in the formation of carbon nanoribbons that are rich in 5-7 defects, i.e., the phagraphene nanoribbon 6 and the TPH-graphene nanoribbon 7. The two different carbon nanoribbons are formed due to the different relative positions of the two fused 2,6-polyazulene chains. These two carbon nanoribbons represent the stripes (colored) cut out from two yet unachieved 2D carbon allotropes, phagraphene $\mathbf{2}$ and TPH-graphene 3. The structures of these two nanoribbons are elucidated unambiguously by scanning tunneling microscopy (STM), non-contact atomic force microscopy (AFM) in ultrahigh vacuum environment at low temperature $(5.2 \mathrm{~K})$ by using $\mathrm{CO}$-functionalized tuning fork sensors, 
and density functional theory (DFT) calculations. The synthetic strategy used in this work may pave the way for the synthesis of 2D extended carbon allotropes with non-benzenoid character.

a

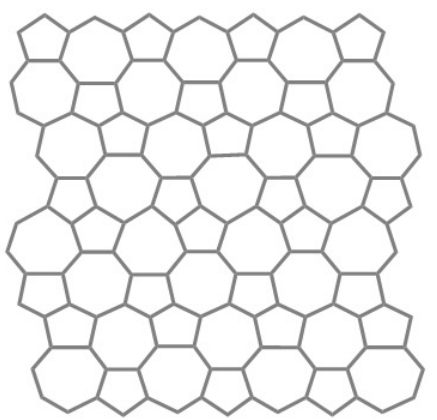

Pentaheptite (5-7) 1 Proposed in 1996 Not achieved

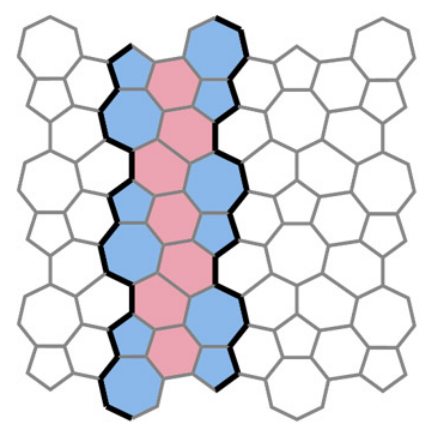

Phagraphene (5-6-7) 2

Proposed in 2015 Nanoribbon achieved in this work

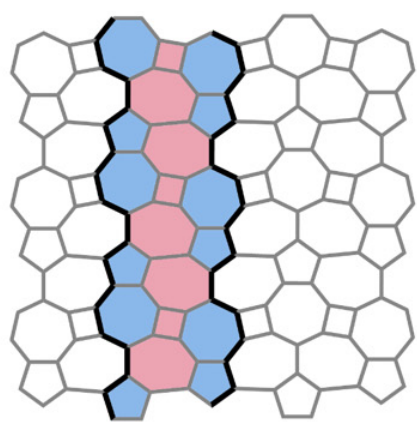

TPH-graphene (4-5-7) 3

Proposed in this work Nanoribbon achieved in this work

b

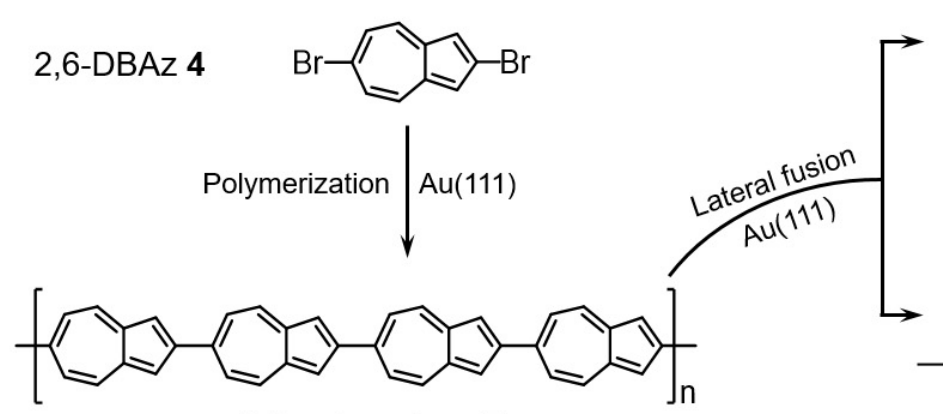

2,6-polyazulene 5

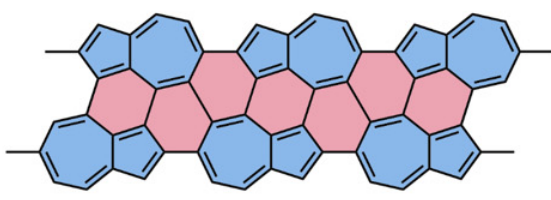

Phagraphene nanoribbon 6

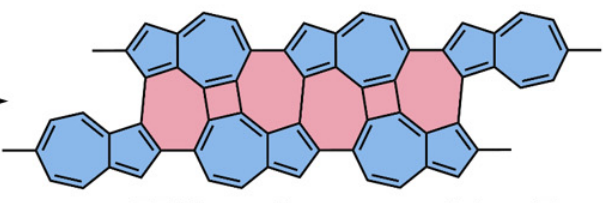

TPH-graphene nanoribbon 7

Figure 1. Reaction scheme for the synthesis of nanoribbons of non-alternant 2D carbon allotropes. a, Skeletons of three proposed 2D carbon allotropes, i.e., pentaheptite 1, phagraphene 2, and THP-graphene 3; all consisting of $\mathrm{sp}^{2}$ carbon atoms. The colored stripes denote the nanoribbons achieved in this work. $\mathbf{b}$, Ullmann C-C coupling of 2,6-dibromoazulene (2,6-DBAz) 4 into 2,6-polyazulene 5 chains and their fusion into carbon nanoribbons rich of non-hexagonal rings, including phagraphene nanoribbon 6 and TPH-graphene nanoribbon 7. These two carbon nanoribbons corresponds to the stripes cut out from the proposed 2D carbon allotropes in (a).

\section{RESULTS}

On-surface synthesis of polyazulene chains. In the first step, polyazulene chains have been prepared on a $\mathrm{Au}(111)$ surface by Ullmann coupling. As shown by Fig. 2a, deposition of a submonolayer of 2,6-DBAz onto $\mathrm{Au}(111)$ at $300 \mathrm{~K}$ followed by two annealing steps over $480 \mathrm{~K}$ to $690 \mathrm{~K}$ leads to ordered domains of chains. The detailed structure of these chains is revealed by a combination of high-resolution STM (Fig. 2b) and constant-height AFM (Fig. 2c) images of a section of the domain (red-framed region in Fig. 2a), using a CO-functionalized tip. ${ }^{40} \mathrm{As}$ can been seen in Fig. $2 \mathrm{~b}$, weak protrusions are observed between the chains, which are assigned as bromine adatoms formed by $\mathrm{C}-\mathrm{Br}$ bond scission. The resulting $\mathrm{Br} \cdots \mathrm{H}$ hydrogen bonds are the driving force for the parallel arrangement of the polyazulene chains, similar to 
previous findings for polyphenylene chains. ${ }^{41}$ The AFM image enables the visualization of the C-C bonds in the azulene units, revealing the feature of annulated 5- and 7-membered rings, in agreement with previous AFM images of single azulene molecules. ${ }^{14}$ Therefore, the molecular structures of these polyazulene chains can be assigned unambiguously, as is exemplified for the left-most chain in Fig. 2c. As can be seen, the azulene units in this chain are not uniformly oriented. The irregularities result from the three different possible types of $\mathrm{C}-\mathrm{C}$ coupling. A uniform, regular orientation would require that the 5membered ring of one azulene unit binds to the 7-membered ring of its neighbor (5-7 coupling). However, 5-5 and 7-7 coupling motifs also occur, as indicated by the labels in Fig 2c. The 7-7 coupling type is associated with characteristic bright features, which can be explained by a nonplanar adsorption conformation due to in-plane steric repulsion between $\mathrm{C}-\mathrm{H}$ bonds of two neighboring azulene units (highlighted with red color). Similar features have been observed previously in AFM images of twisted phenyl units or aliphatic hydrocarbons ${ }^{42-45}$ In contrast, for the 5-7 and 5-5 coupling motifs, the involved azulene units exhibit almost planar adsorption configurations, indicating reduced repulsion due to the larger distances between the $\mathrm{C}-\mathrm{H}$ bonds (Fig. 2c, colored blue and yellow).
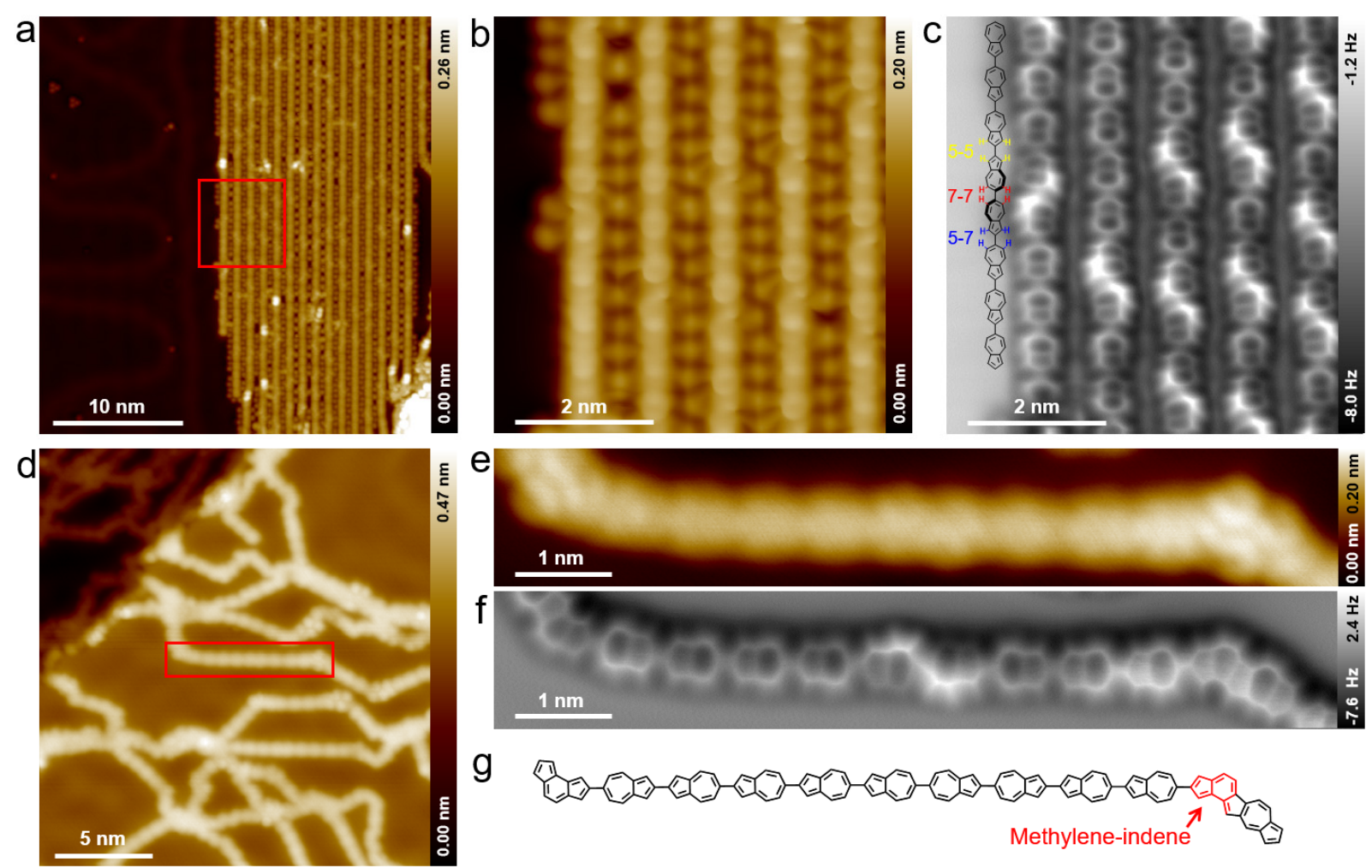

Figure 2. STM and nc-AFM images of polyazulene chains on Au(111). a,d, Overview STM images of polyazulene chains formed after deposition of a submonolayer of 2,6-DBAz onto $\mathrm{Au}(111)$ at $300 \mathrm{~K}$ followed by successive annealing to $480 \mathrm{~K}$ for 30 minutes and $690 \mathrm{~K}$ for 20 minutes (a), and $730 \mathrm{~K}$ for 30 minutes (d). b,c, Zoom-in STM (b) and constant-height AFM (c) images of the red-framed region in panel (a). The chemical structure of the left-most chain in panel (c) highlights the different types of C-C coupling (5-7, 5-5, and 7-7). e-g, Zoom-in STM (e), constant-height AFM (f) images and chemical structure (g) of the curved chain in the red framed region of panel (d). The methylene-indene units formed by isomerization of azulene units has been colored red in $(\mathbf{g})$. Image parameters: (a),(e) $U=10 \mathrm{mV}, I=10 \mathrm{pA}$; (b) $U=10 \mathrm{mV}, I=70 \mathrm{pA}$; (d) $U=100 \mathrm{mV}, I=10 \mathrm{pA}$. Tip distances 
for the constant-height AFM images: (c) $z=50 \mathrm{pm}$, and (f) $z=25 \mathrm{pm}$ with respect to the tunneling gap at an STM set point of $I=10 \mathrm{pA}$ and $U=10 \mathrm{mV}$ above the Au(111) substrate. All the STM and AFM images are measured with a CO-functionalized tip and an oscillation amplitude of $70 \mathrm{pm}$.

Further annealing of the sample in Figure $2 \mathrm{a}$ to $730 \mathrm{~K}$ results in the separation and occasional bending of the polyazulene chains, as shown by the STM image in Figure $2 \mathrm{~d}$. In addition, a certain amount of single chains cross-link at their terminals. These structural changes can be interpreted as follows. Annealing to $730 \mathrm{~K}$ desorbs the bromine adatoms from $\mathrm{Au}(111)$. This is evidenced by the absence of the weak protrusions around the chains. As a result, the chains have an increased probability to encounter and react through dehydrogenative coupling. Note that the $\mathrm{C}-\mathrm{H}$ bond scission on $\mathrm{Au}(111)$ surface at $730 \mathrm{~K}$ is expected according to previous work. ${ }^{46}$ The bending of the polyazulene chains is caused by rearrangements in the carbon framework, as is demonstrated for a typical single chain with two bends (red-framed region in Fig. 2d). Its structure has been scrutinized by detailed STM (Fig. 2e) and AFM (Fig. 2f) measurements and is shown in Fig. 2g. The straight part of this chain consists entirely of azulene units, whereas the bend bears a methylene-indene moiety, as marked by red color. Since methylene-indene is an isomer of azulene, it is likely that an azulene moiety undergoes carbon rearrangement (or isomerization) into the methylene-indene moiety on $\mathrm{Au}(111)$ already at $730 \mathrm{~K}$. Besides the methylene-indene motif, naphthalene as another isomer of azulene has occasionally been observed as a structural motif at the corners of a single curved chain, as shown by Figure S1. Related carbon rearrangements have also been reported for a polycyclic aromatic hydrocarbon with azulene moieties on a $\mathrm{Cu}(001)$ surface. ${ }^{34}$

Lateral fusion of polyazulene chains into non-alternant carbon nanoribbons. At this stage (after heating to $730 \mathrm{~K}$, see Fig.2d) also pairwise lateral dehydrogenative fusion of the polyazulene chains is induced, leading to the formation of wider nanoribbons as presented by Fig. 3a and 3e showing different sample regions. Two representative nanoribbons are selected for detailed structural analysis. The structure of the nanoribbon in the red-framed region in Fig. $3 a$ is revealed by the zoom-in STM (Fig. 3b) and the corresponding bond-resolving AFM (Fig. 3c) images. As illustrated in Fig. 3d, the nanoribbon contains two chains (blue colored) that are fully fused forming polygons (red colored) in the interspace. The major structural motif in the chains (blue) is azulene. Minority structural motifs are naphthalene (marked by blue arrows) and methylene-indene moieties (marked by red arrows), which originate from the isomerization of the azulene moieties in the polyazulene chains as discussed above. A short section of the nanoribbons marked by the red bracket in Fig. $3 \mathrm{~d}$ represents the perfect lateral fusion of two oppositely oriented, regular 2,6-polyazulene chains. As can be seen, hexagons are formed exclusively between the two chains. Therefore, this structure can be considered as a stripe cut out from the carbon-allotrope phagraphene proposed in previous theoretical work. ${ }^{25}$ Another nanoribbon with different structure is marked by the red frame in Fig. 3e; its enlargement is shown in Fig. 3f. The detailed structure of this ribbon, as derived from the AFM image in Fig. 3g, is displayed in Fig. $3 \mathrm{~h}$. As can be seen, this nanoribbon is again based on two single chains consisting of $\mathrm{C}-\mathrm{C}$ bonded azulene (and methylene-indene) moieties. Similar to the first nanoribbon (Fig. $3 \mathrm{C}$ ), the section marked by the red bracket shows a periodic structure derived from two oppositely oriented regular 2,6-polyazulene chains. Compared to the first case (Fig. 3d), the two chains have a shifted relative position, such that a carbon nanoribbon with four, five, and sevenmembered rings is obtained. Thus, this nanoribbon represents a stripe of the TPH-graphene in Fig. 1a. Additional examples of these two types of non-alternant carbon nanoribbons are shown in Fig. S1. 

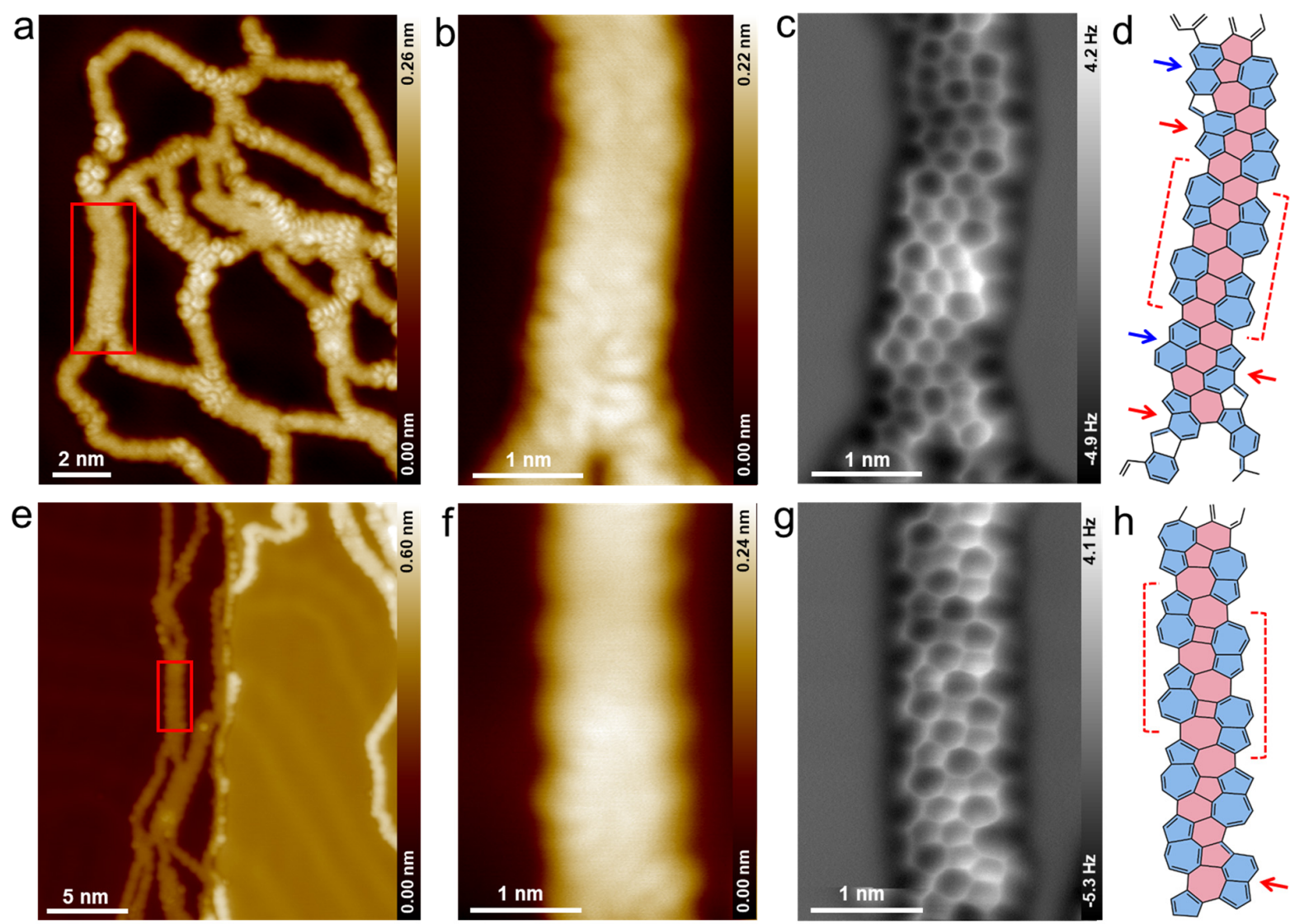

Figure 3. STM and nc-AFM images of carbon nanoribbons on Au(111). a,e, Overview STM images of different regions of the sample (same as in Fig. 2d), showing wider nanoribbons with different structures. b-d, High resolution STM (b), constant-height AFM (c) images and chemical structure (d) of the nanoribbon in the redframed region in panel (a). f-h, High resolution STM (f), constant-height AFM (g) images and chemical structure (h) of the nanoribbon in the red-framed region in panel (e). In (d,h), the blue color marks the two chains fused into the nanoribbon by forming polygons marked by red color. Blue and red arrows point out the naphthalene and methylene-indene moieties, respectively, formed by isomerization of azulene moieties. Image parameters: (a),(b) $U=10 \mathrm{mV}, I=10 \mathrm{pA}$; (e) (f) $U=100 \mathrm{mV}, I=10 \mathrm{pA}$. Tip distances for the AFM images: (c) $z=120 \mathrm{pm}$, and (g) $z=80 \mathrm{pm}$ with respect to the STM set point of $I=10 \mathrm{pA}$ and $U=10 \mathrm{mV}$ above the $\mathrm{Au}(111)$ substrate. All the STM and AFM images are measured with a CO-functionalized tip and an oscillation amplitude of $70 \mathrm{pm}$.

\section{DISCUSSION}

The high-yield formation of longer phagraphene 6 and TPH-graphene $\mathbf{7}$ nanoribbons is still challenging, mainly because of two issues: First, carbon allotropes with non-alternant structural elements may isomerize to their corresponding alternant structures, which are usually more stable. In particular, isomerization of the azulene units in $\mathbf{6}$ and $\mathbf{7}$ to naphthalene units is expected to be energetically favorable, considering that naphthalene is $1.62 \mathrm{eV}$ more stable than azulene. ${ }^{47}$ If $\mathrm{C}-\mathrm{C}$ bond rearrangements are kinetically possible under the reaction conditions, these transformation are likely to occur, as shown in Fig. S2. The stability of the non-alternant structures is therefore an important aspect of their on-surface synthesis. Indeed, the present periodic DFT calculations for the free-standing, planar nanoribbons (cf. Figure 4) suggest that 6 (Fig. 4a) is thermodynamically less stable by $2.7 \mathrm{eV}$ per $\mathrm{C}_{20} \mathrm{H}_{6}$ formula unit than its 
alternant counterpart $\mathbf{6}$ ' shown in Fig. $4 \mathrm{~b}$. Both structures enclosing four-membered rings (7 and $\mathbf{7}$ ', Fig. $4 c, d)$ are even more destabilized by 5.2 and $7.2 \mathrm{eV}$, respectively, compared to the low-energy structure $\mathbf{6}^{\prime}$. This may be rationalized based on the high ring tension within the four-membered rings in $\mathbf{7}$ and $\mathbf{7}^{\prime}$. The hypothetical naphthalene-based nanoribbon $\mathbf{7}^{\prime}$ also features an exceptionally long carbon-carbon bond of $179 \mathrm{pm}$, indicating a highly strained structure.

In the following, we discuss that the relative stabilities of the different structures are also consistent with qualitative aromaticity considerations. The color code of the bonds in Fig. 4 shows the calculated bond lengths for the two nanoribbons $\mathbf{6}$ and $\mathbf{7}$ (Fig. 4a,c) and for the corresponding hypothetical structures $\mathbf{6}^{\prime}$ and $\mathbf{7}^{\prime}$ formed by isomerization of their azulene units to naphthalene units (Fig. $4 \mathrm{~b}, \mathrm{~d}$ ). Based on the C-C bond lengths, the aromatic character of the rings can be assessed using the harmonic oscillator model of aromaticity (HOMA). ${ }^{48} \mathrm{~A}$ higher HOMA value is generally associated with increased aromatic stabilization. ${ }^{48}$ The HOMA values of the rings are illustrated by the different shades of orange color in Fig. 4. Comparison of the phagraphene ribbon $\mathbf{6}$ with its alternant isomer $\mathbf{6}^{\prime}$ reveals that the latter has considerably higher HOMA values and thus experiences increased aromatic stabilization. Therefore, isomerization of $\mathbf{6}$ to its alternant counterpart $\mathbf{6}$ ' should be energetically favored, in agreement with the experimental observations (Fig. S2) and the DFT energies $\left(\Delta E_{6^{\prime}-6}=-2.7 \mathrm{eV}\right)$. In contrast, the HOMA values indicate that the $\mathrm{TPH}$-graphene nanoribbon $\mathbf{7}$ is more stable than its hypothetical isomerization product $7^{\prime}$, again in line with the DFT results $\left(\Delta E_{7^{\prime}-7}=2.0 \mathrm{eV}\right)$. Comparison of the HOMA data for the two experimental nanoribbons $\mathbf{6}$ and $\mathbf{7}$ reveals a slight advantage in aromatic stabilization for $\mathbf{6}$, in agreement with the difference of their DFT energies $\left(\Delta E_{7-6}=2.5 \mathrm{eV}\right)$. According to these considerations, 7 may be more resistant to isomerization of azulene to naphthalene units than 6 .

a

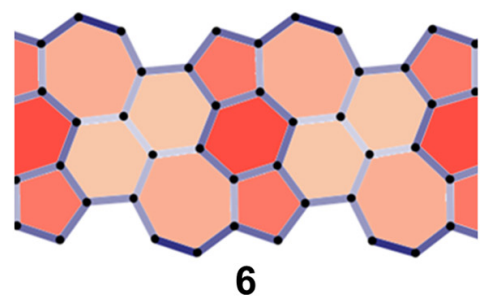

C

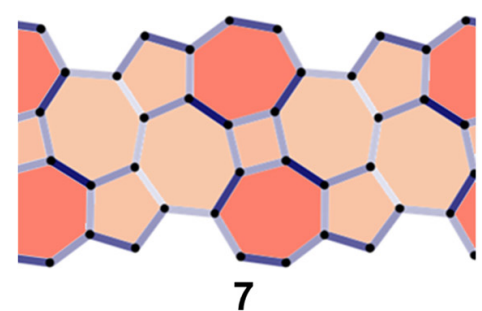

b

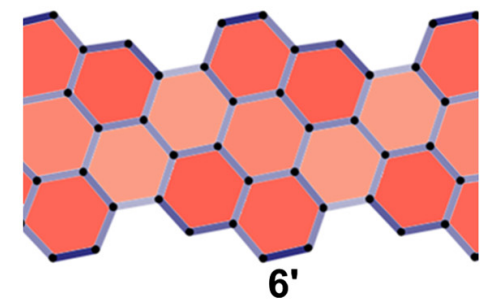

d

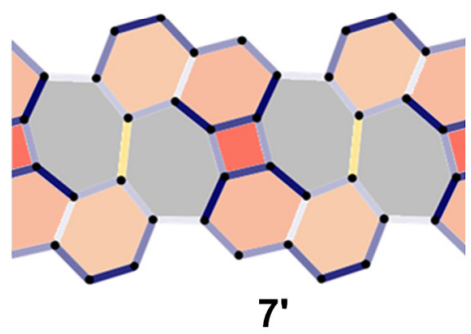

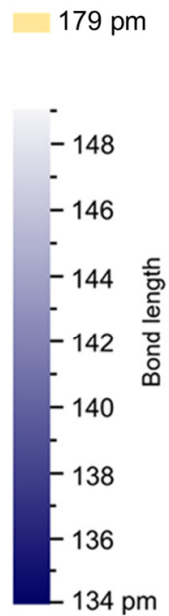

$134 \mathrm{pm}$

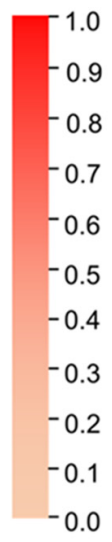

$-5.8$

Figure 4. DFT-calculated C-C bond lengths of planar carbon nanoribbons. a,c, Optimized gas-phase chemical structure of the non-alternant phagraphene nanoribbon $\mathbf{6}$ (a) and TPH-graphene nanoribbon $\mathbf{7}$ (c). b,d, GNR counterparts of phagraphene and TPH-graphene nanoribbons obtained by isomerization of azulene moieties into naphthalene in these two types of nanoribbons ( $\mathbf{6}^{\prime}$ and $\left.\mathbf{7}^{\prime}\right)$. The bond lengths and harmonic oscillator model of aromaticity (HOMA) values of each polygon are presented with gradient colors as illustrated by the color scales at the right part of the figure. The average HOMA values are (a) 0.426 , (b) 0.614 , (c) 0.282 , (d) -1.48 . 
Second, the formation of both phagraphene and TPH-graphene nanoribbons require the lateral fusion of 2,6-polyazulene chains with exclusive 5-7 connections and their alignment along opposite orientations. Such condition is demanding considering that the polyazulene chains are formed with randomly orientated azulene units at the first Ullmann coupling step. This is also the reason why wider nanoribbons with more than two fused chains have only rarely been observed: They require alternating orientations of the 2,6-polyazulene chains, which is of diminishing probability with the increasing width of the ribbon.

\section{CONCLUSION}

In conclusion, different types of carbon nanoribbons with non-alternant topology of the $\pi$-electron system, i.e., the phagraphene nanoribbon 6 and the THP-graphene nanoribbon 7, have been achieved by the lateral dehydrogenative coupling of polyazulene chains formed in a preceding step via surface Ullmann coupling of the 2,6-dibromoazulene precursor on $\mathrm{Au}(111)$. The different structures of these nanoribbons are determined by the relative positions of the fused polyazulene chains. Their yields are limited by the mixed connection types and isomerization of the azulene units in the polyazulene chains. These nonalternant carbon nanoribbons may represent promising candidates for the search of new carbon-based quantum materials with exotic properties. Moreover, the hierarchical coupling of non-hexagonal conjugated rings of carbon atoms opens the possibilities for the synthesis of novel $2 \mathrm{D}$ carbon allotropes.

\section{METHODS}

Synthesis: 2,6-Dibromoazulene was prepared by using a published procedure as described in the Supplementary Information part 2. The details of the synthesis and characterization of 2,6dibromoazulene are also included in the Supplementary Information part 2.

Sample preparation: The $\mathrm{Au}(111)$ crystal (MaTeck, Germany) was cleaned by multiple cycles of $\mathrm{Ar}^{+}$ sputtering $\left(E=1.5 \mathrm{kV}, \mathrm{I}=3.6 \mu \mathrm{A}, \mathrm{p}=6 \times 10^{-6} \mathrm{mbar}\right.$ for initial cycles and $E=0.8 \mathrm{kV}, \mathrm{I}=1.1 \mu \mathrm{A}, \mathrm{p}=3 \times 10^{-6}$ mbar for final cycles) and annealing (1000 K for initial cycles and $730 \mathrm{~K}$ for final cycles). The 2,6-DBAz molecules were evaporated on the $\mathrm{Au}(111)$ surface by using a home-built evaporation device held at room temperature. ${ }^{49}$ To induce chemical reactions on the surface, the sample was placed in a heating station (Scienta Omicron, Germany) where it was heated by thermal radiation of a tungsten filament. The sample temperatures were deduced from the heating power of the filament and a thermocouple that is attached to the heating station close to the sample plate. The heating station was previously calibrated by using a second thermocouple that was spot-welded to the center of an empty sample plate.

STM/AFM: STM and AFM images were obtained at $5.2 \mathrm{~K}$ and a pressure below $1 \times 10^{-10} \mathrm{mbar}$ with a low temperature AFM/STM (Scienta Omicron, Germany). A Q-plus tuning fork sensor with a tungsten tip was used. The tip was sharpened by voltage pulses and indentations into the Au(111) surface. The AFM tip was functionalized with a $\mathrm{CO}$ molecule using standard procedures described in the literature..$^{50}$ The resonance frequency and quality factor of the sensor $\left(f_{r}=25.866 \mathrm{kHz}, Q=18000-25000\right)$ was determined by acoustic tunes with a tip-sample separation of about $10 \mathrm{~nm}$. The Q-plus sensor was driven in frequency modulation mode using an external phase-locked loop electronics (MFLI, Zürich Instruments, Switzerland) for obtaining both STM and AFM images ${ }^{45}$ The oscillation amplitude of the sensor was kept constant at $70 \mathrm{pm}$. AFM images were taken in constant-height and constant current-scanning modes. ${ }^{45}$

Computational details: Periodic density functional-theory calculations of free-standing carbon nanoribbons were performed with the Vienna Ab Initio Simulation Package (VASP) using the PBE functional and the D3(BJ) dispersion correction. Please see the SI for further details. 


\section{ASSOCIATED CONTENT}

Supporting Information. The Supporting Information is available free of charge on the ACS Publications website. Precursor synthesis and characterization, additional STM and AFM images, and details of the DFT calculations.

\section{AUTHOR INFORMATION}

\section{Corresponding Authors}

* daniel.ebeling@ap.physik.uni-giessen.de

* gerhard.hilt@uni-oldenburg.de

*wolfgang.hieringer@fau.de

*michael.gottfried@chemie.uni-marburg.de

\section{Author Contributions}

$\ddagger$ Q.F. and D.M.J. contributed equally.

\section{Notes}

The authors declare no competing financial interest.

\section{ACKNOWLEDGMENT}

Financial support by the Deutsche Forschungsgemeinschaft via the grants GO 1812/2-1, HI 655/18-1 and EB535/1-1, 223848855-SFB 1083, the Cluster of Excellence EXC 315 "Engineering of Advanced Materials", and the GRK (Research Training Group) 2204 "Substitute Materials for Sustainable Energy Technologies" is gratefully acknowledged. The RRZE of the University of Erlangen-Nürnberg is acknowledged for computational resources. Q.T.F thanks the Alexander von Humboldt-Foundation for a Research Fellowship for Postdoctoral Researchers.

\section{REFERENCES:}

1. Talirz, L.; Ruffieux, P.; Fasel, R. On-Surface Synthesis of Atomically Precise Graphene Nanoribbons. Adv. Mater. 2016, 28, 6222-31.

2. Ruffieux, P.; Wang, S.; Yang, B.; Sánchez-Sánchez, C.; Liu, J.; Dienel, T.; Talirz, L.; Shinde, P.; Pignedoli, C. A.; Passerone, D.; Dumslaff, T.; Feng, X.; Müllen, K.; Fasel, R. On-Surface Synthesis of Graphene Nanoribbons with Zigzag Edge Topology. Nature 2016, 531, 489-492.

3. Rizzo, D. J.; Veber, G.; Cao, T.; Bronner, C.; Chen, T.; Zhao, F.; Rodriguez, H.; Louie, S. G.; Crommie, M. F.; Fischer, F. R. Topological Band Engineering of Graphene Nanoribbons. Nature 2018, 560, 204-208.

4. Gröning, O.; Wang, S.; Yao, X.; Pignedoli, C. A.; Borin Barin, G.; Daniels, C.; Cupo, A.; Meunier, V.; Feng, X.; Narita, A.; Müllen, K.; Ruffieux, P.; Fasel, R. Engineering of Robust Topological Quantum Phases in Graphene Nanoribbons. Nature 2018, 560, 209-213.

5. $\quad$ Cai, J.; Ruffieux, P.; Jaafar, R.; Bieri, M.; Braun, T.; Blankenburg, S.; Muoth, M.; Seitsonen, A. P.; Saleh, M.; Feng, X.; Müllen, K.; Fasel, R. Atomically Precise Bottom-up Fabrication of Graphene Nanoribbons. Nature 2010, 466, 470-473.

6. Moreno, C.; Vilas-Varela, M.; Kretz, B.; Garcia-Lekue, A.; Costache, M. V.; Paradinas, M.; Panighel, M.; Ceballos, G.; Valenzuela, S. O.; Peña, D.; Mugarza, A. Bottom-Up Synthesis of Multifunctional Nanoporous Graphene. Science 2018, 360, 199. 
7. Bieri, M.; Treier, M.; Cai, J.; Ait-Mansour, K.; Ruffieux, P.; Gröning, O.; Gröning, P.; Kastler, M.; Rieger, R.; Feng, X.; Müllen, K.; Fasel, R. Porous Graphenes: Two-Dimensional Polymer Synthesis with Atomic Precision. Chem. Commun. 2009, 6919-6921.

8. Fan, Q.; Wang, C.; Liu, L.; Han, Y.; Zhao, J.; Zhu, J.; Kuttner, J.; Hilt, G.; Gottfried, J. M. Covalent, Organometallic, and Halogen-Bonded Nanomeshes from Tetrabromo-Terphenyl by SurfaceAssisted Synthesis on Cu(111). J. Phys. Chem. C 2014, 118, 13018-13025.

9. Kolmer, M.; Zuzak, R.; Steiner, A. K.; Zajac, L.; Engelund, M.; Godlewski, S.; Szymonski, M.; Amsharov, K. Fluorine-Programmed Nanozipping to Tailored Nanographenes on Rutile $\mathrm{TiO}_{2}$ Surfaces. Science 2019, 363, 57-60.

10. Treier, M.; Pignedoli, C. A.; Laino, T.; Rieger, R.; Müllen, K.; Passerone, D.; Fasel, R. SurfaceAssisted Cyclodehydrogenation Provides a Synthetic Route Towards Easily Processable and Chemically Tailored Nanographenes. Nat. Chem. 2011, 3, 61-67.

11. Hieulle, J.; Carbonell-Sanroma, E.; Vilas-Varela, M.; Garcia-Lekue, A.; Guitian, E.; Pena, D.; Pascual, J. I. On-Surface Route for Producing Planar Nanographenes with Azulene Moieties. Nano lett. 2018, $18,418-423$.

12. Beser, U.; Kastler, M.; Maghsoumi, A.; Wagner, M.; Castiglioni, C.; Tommasini, M.; Narita, A.; Feng, X.; Müllen, K. A C216-Nanographene Molecule with Defined Cavity as Extended Coronoid. J. Am. Chem. Soc. 2016, 138, 4322-4325.

13. Zhong, Q.; Hu, Y.; Niu, K.; Zhang, H.; Yang, B.; Ebeling, D.; Tschakert, J.; Cheng, T.; Schirmeisen, A.; Narita, A.; Müllen, K.; Chi, L. Benzo-Fused Periacenes or Double Helicenes? Different Cyclodehydrogenation Pathways on Surface and in Solution. J. Am. Chem. Soc. 2019, 141, 73997406.

14. Klein, B. P.; van der Heijden, N. J.; Kachel, S. R.; Franke, M.; Krug, C. K.; Greulich, K. K.; Ruppenthal, L.; Müller, P.; Rosenow, P.; Parhizkar, S.; Bocquet, F. C.; Schmid, M.; Hieringer, W.; Maurer, R. J.; Tonner, R.; Kumpf, C.; Swart, I.; Gottfried, J. M. Molecular Topology and the Surface Chemical Bond: Alternant Versus Nonalternant Aromatic Systems as Functional Structural Elements. Phys. Rev. X 2019, 9, 011030.

15. Georgakilas, V.; Perman, J. A.; Tucek, J.; Zboril, R. Broad Family of Carbon Nanoallotropes: Classification, Chemistry, and Applications of Fullerenes, Carbon Dots, Nanotubes, Graphene, Nanodiamonds, and Combined Superstructures. Chem. Rev. 2015, 115, 4744-4822.

16. Sui, C.; Zhao, Y.; Zhang, Z.; He, J.; Zhang, Z.; He, X.; Wang, C.; Wu, J. Morphology-Controlled Tensile Mechanical Characteristics in Graphene Allotropes. ACS Omega 2017, 2, 3977-3988.

17. Enyashin, A. N.; Ivanovskii, A. L. Graphene Allotropes. Phys. Status Solidi B 2011, 248, 1879-1883.

18. Molepo, M. P.; Mapasha, R. E.; Obodo, K. O.; Chetty, N. First Principles Calculations of Pentaheptite Graphene and Boronitrene Derivatives. Computat. Mater. Sci. 2014, 92, 395-400.

19. Fthenakis, Z. G.; Lathiotakis, N. N. Graphene Allotropes Under Extreme Uniaxial Strain: An ab initio Theoretical Study. Phys. Chem. Chem. Phys. 2015, 17, 16418-16427.

20. Crespi, V. H.; Benedict, L. X.; Cohen, M. L.; Louie, S. G. Prediction of a Pure-Carbon Planar Covalent Metal. Phys. Rev. B 1996, 53, 13303-13305. 
21. Terrones, H.; Terrones, M.; Hernández, E.; Grobert, N.; Charlier, J. C.; Ajayan, P. M. New Metallic Allotropes of Planar and Tubular Carbon. Phys. Rev. Lett. 2000, 84, 1716-1719.

22. Liu, Y.; Wang, G.; Huang, Q.; Guo, L.; Chen, X. Structural and Electronic Properties of T Graphene: A Two-Dimensional Carbon Allotrope with Tetrarings. Phys. Rev. Lett. 2012, 108, 225505.

23. Su, C.; Jiang, H.; Feng, J. Two-Dimensional Carbon Allotrope with Strong Electronic Anisotropy. Phys. Rev. B 2013, 87, 075453.

24. Sharma, B. R.; Manjanath, A.; Singh, A. K. Pentahexoctite: A New Two-Dimensional Allotrope of Carbon. Sci. Rep. 2014, 4, 7164.

25. Wang, Z.; Zhou, X. F.; Zhang, X.; Zhu, Q.; Dong, H.; Zhao, M.; Oganov, A. R. Phagraphene: A LowEnergy Graphene Allotrope Composed of 5-6-7 Carbon Rings with Distorted Dirac Cones. Nano lett. 2015, 15, 6182-6186.

26. Červenka, J.; Flipse, C. F. J. Structural and Electronic Properties of Grain Boundaries in Graphite: Planes of Periodically Distributed Point Defects. Phys. Rev. B 2009, 79, 195429.

27. Peres, N. M. R.; Guinea, F.; Castro Neto, A. H. Electronic Properties of Disordered TwoDimensional Carbon. Phys. Rev. B 2006, 73, 125411.

28. Červenka, J.; Katsnelson, M. I.; Flipse, C. F. J. Room-Temperature Ferromagnetism in Graphite Driven by Two-Dimensional Networks of Point Defects. Nat. Phys. 2009, 5, 840.

29. Malola, S.; Häkkinen, H.; Koskinen, P. Structural, Chemical, and Dynamical Trends in Graphene Grain Boundaries. Phys. Rev. B 2010, 81, 165447.

30. Grantab, R.; Shenoy, V. B.; Ruoff, R. S. Anomalous Strength Characteristics of Tilt Grain Boundaries in Graphene. Science 2010, 330, 946-948.

31. Wei, Y.; Wu, J.; Yin, H.; Shi, X.; Yang, R.; Dresselhaus, M. The Nature of Strength Enhancement and Weakening by Pentagon-Heptagon Defects in Graphene. Nat. Mater. 2012, 11, 759.

32. Banhart, F.; Kotakoski, J.; Krasheninnikov, A. V. Structural Defects in Graphene. ACS Nano 2011, 5, 26-41.

33. Cockayne, E.; Rutter, G. M.; Guisinger, N. P.; Crain, J. N.; First, P. N.; Stroscio, J. A. Grain Boundary Loops in Graphene. Phys. Rev. B 2011, 83, 195425.

34. Shiotari, A.; Nakae, T.; Iwata, K.; Mori, S.; Okujima, T.; Uno, H.; Sakaguchi, H.; Sugimoto, Y. StrainInduced Skeletal Rearrangement of a Polycyclic Aromatic Hydrocarbon on a Copper Surface. Nat. Commun. 2017, 8, 16089.

35. Mishra, S.; Krzeszewski, M.; Pignedoli, C. A.; Ruffieux, P.; Fasel, R.; Gryko, D. T. On-Surface Synthesis of a Nitrogen-Embedded Buckybowl with Inverse Stone-Thrower-Wales Topology. Nat. Commun. 2018, 9, 1714.

36. Mishra, S.; Lohr, T. G.; Pignedoli, C. A.; Liu, J.; Berger, R.; Urgel, J. I.; Müllen, K.; Feng, X.; Ruffieux, P.; Fasel, R. Tailoring Bond Topologies in Open-Shell Graphene Nanostructures. ACS Nano 2018, 12, 11917-11927. 
37. Liu, M.; Liu, M.; She, L.; Zha, Z.; Pan, J.; Li, S.; Li, T.; He, Y.; Cai, Z.; Wang, J.; Zheng, Y.; Qiu, X.; Zhong, D. Graphene-Like Nanoribbons Periodically Embedded with Four- and Eight-Membered Rings. Nat. Commun. 2017, 8, 14924.

38. Liu, M.; Liu, M.; Zha, Z.; Pan, J.; Qiu, X.; Li, T.; Wang, J.; Zheng, Y.; Zhong, D. Thermally Induced Transformation of Nonhexagonal Carbon Rings in Graphene-like Nanoribbons. J. Phys. Chem. $C$ 2018, 34, 1884-1891.

39. Ruedenberg, K. Quantum Mechanics of Mobile Electrons in Conjugated Bond Systems. III. Topological Matrix as Generatrix of Bond Orders. J. Chem. Phys. 1961, 34, 1884-1891.

40. Gross, L.; Mohn, F.; Moll, N.; Liljeroth, P.; Meyer, G. The Chemical Structure of a Molecule Resolved by Atomic Force Microscopy. Science 2009, 325, 1110.

41. Wang, W. H.; Shi, X. Q.; Wang, S. Y.; Van Hove, M. A.; Lin, N. Single-Molecule Resolution of an Organometallic Intermediate in a Surface-Supported Ullmann Coupling Reaction. J. Am. Chem. Soc. 2011, 133, 13264-13267.

42. Ebeling, D.; Sekutor, M.; Stiefermann, M.; Tschakert, J.; Dahl, J. E. P.; Carlson, R. M. K.; Schirmeisen, A.; Schreiner, P. R. Assigning the Absolute Configuration of Single Aliphatic Molecules by Visual Inspection. Nat. Commun. 2018, 9, 2420.

43. Ebeling, D.; Sekutor, M.; Stiefermann, M.; Tschakert, J.; Dahl, J. E. P.; Carlson, R. M. K.; Schirmeisen, A.; Schreiner, P. R. London Dispersion Directs On-Surface Self-Assembly of [121]Tetramantane Molecules. ACS Nano 2017, 11, 9459-9466.

44. Ebeling, D.; Zhong, Q.; Schloder, T.; Tschakert, J.; Henkel, P.; Ahles, S.; Chi, L.; Mollenhauer, D.; Wegner, H. A.; Schirmeisen, A. Adsorption Structure of Mono- and Diradicals on a Cu(111) Surface: Chemoselective Dehalogenation of 4-Bromo-3"-iodo-p-terphenyl. ACS Nano 2019, 13, 324-336.

45. Martin-Jimenez, D.; Ahles, S.; Mollenhauer, D.; Wegner, H. A.; Schirmeisen, A.; Ebeling, D. BondLevel Imaging of the 3D Conformation of Adsorbed Organic Molecules Using Atomic Force Microscopy with Simultaneous Tunneling Feedback. Phys. Rev. Lett. 2019, 122, 196101.

46. Basagni, A.; Sedona, F.; Pignedoli, C. A.; Cattelan, M.; Nicolas, L.; Casarin, M.; Sambi, M. Molecules-Oligomers-Nanowires-Graphene Nanoribbons: A Bottom-Up Stepwise On-Surface Covalent Synthesis Preserving Long-Range Order. J. Am. Chem. Soc. 2015, 137, 1802-1808.

47. Roth, W. R.; Böhm, M.; Lennartz, H.-W.; Vogel, E. Resonance Energy of Bridged [10]Annulenes. Angew. Chem. Int. Ed. 1983, 22, 1007-1008.

48. Setiawan, D.; Kraka, E.; Cremer, D. Quantitative Assessment of Aromaticity and Antiaromaticity Utilizing Vibrational Spectroscopy. J. Org. Chem. 2016, 81, 9669-9686.

49. Zint, S.; Ebeling, D.; Ahles, S.; Wegner, H. A.; Schirmeisen, A. Subsurface-Controlled Angular Rotation: Triphenylene Molecules on Au(111) Substrates. J. Phys. Chem. C 2016, 120, 1615-1622.

50. Bartels, L.; Meyer, G.; Rieder, K. H. Controlled Vertical Manipulation of Single CO Molecules with the Scanning Tunneling Microscope: A Route to Chemical Contrast. Appl. Phys. Lett. 1997, 71, 213215. 
TOC Graphic

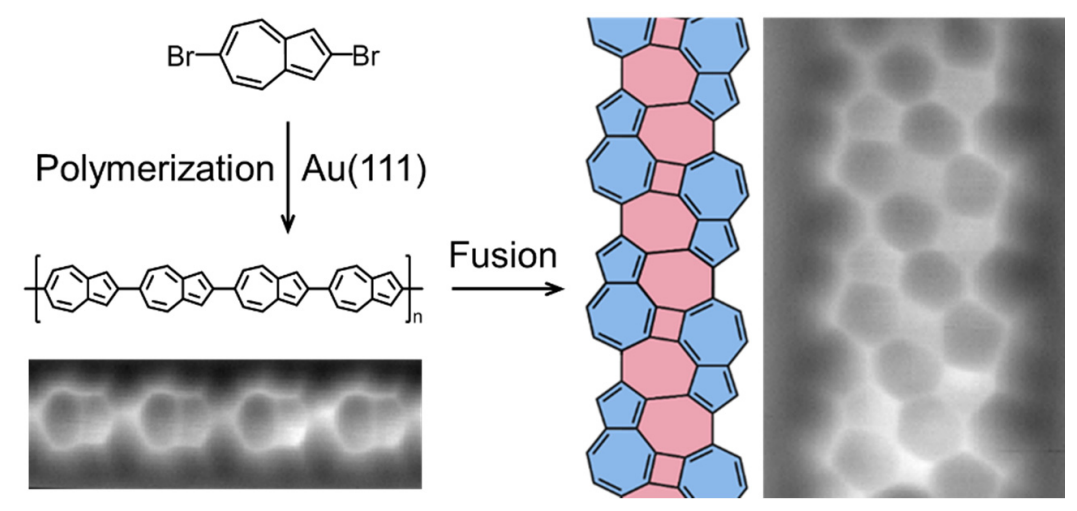

